Ameloblastic fibro-odontoma (AFO) is an uncommon, benign, mixed odontogenic tumour occurring predominantly in children and adolescents. The purpose of this study was to report an unusual case of AFO in the mandible of a five-year-old male patient showing evidence of chondroid tissue formation. Clinical, radiographic, histopathological and immunohistochemical findings were presented. Intraoral examination revealed a swelling extending from the right deciduous lateral incisor to the left second molar area. Computed tomography (CT) showed a large, well-demarcated multilocular radiolucency with radiopaque deposits. Histologically, the lesion was composed of connective tissue stroma resembling the dental papilla, with epithelial islands, and amorphous masses of enamel and dentin consistent with a diagnosis of AFO, as well as, evidence of large areas showing cartilaginous tissue formation. Immunohistochemistry using cytokeratin, vimentin and s-100 were helpful in determining the origin of the different odontogenic tissues. Surgical excision of the lesion was performed. The patient has been monitored and the lesion has not recurred. We report the first case of AFO showing an unusual behaviour of chondroid tissue formation.

Key words: odontogenic tumours, ameloblastic fibro-odontoma, chondroid tissue.

Contemp Oncol (Pozn) 2018; 22 (1): 50-53 DOI: https://doi.org/10.5114/wo.2018.74395

\section{Ameloblastic fibro-odontoma with chondroid tissue formation}

\author{
Noura Aly ${ }^{1}$, Hatem Amer², Omar El Khatib²
}

${ }^{1}$ Faculty of Dentistry, Cairo University, Egypt

2Department of Oral and Maxillofacial Pathology, Faculty of Dentistry, Cairo University, Egypt

\section{Introduction}

Ameloblastic fibroma (AF) is a rare mixed tumour characterised by the neoplastic proliferation of both epithelial and mesenchymal components but without formation of either dentin or enamel. Lesions having similar components, in addition to dentin alone or dentin and enamel, are termed ameloblastic fibrodentinoma (AFD) and ameloblastic fibro-odontoma (AFO) [1].

Ameloblastic fibroma and AFO are defined, according to the recent World Health Organisation classification of Odontogenic Tumours published in 2017, as lesions with odontogenic epithelium and odontogenic ectomesenchyme, with or without hard tissue formation) [1]

Ameloblastic fibroma and AFO are usually found in young adults, with males showing slightly higher prevalence in cases of AF, but no significant gender predilection was observed in AFO. Failure of tooth eruption and swelling are usually associated with such lesions. Clinically, they present as an asymptomatic swelling affecting the posterior aspect of the mandible. Radiographs show a well-defined radiolucent area, whereas AFO may show radiopaque material of variable size and form [2].

The aim of this paper is to report an unprecedented case of ameloblastic fibro-odontome demonstrating an unusual behaviour of cartilage like tissue formation. Clinical, radiographic, and histopathological features are noted.

\section{Case report}

A 5-year-old male child patient was referred to the Department of Oral and Maxillofacial Surgery, Faculty of Dentistry, Cairo University with a complaint of an asymptomatic slowly growing swelling in the left mandibular area of 8 months duration. The medical and family histories were unremarkable, as were the results of the physical examination.

Extraoral examination revealed facial asymmetry, with swelling present on the left side of the mandible. Intraoral examination revealed a non-tender

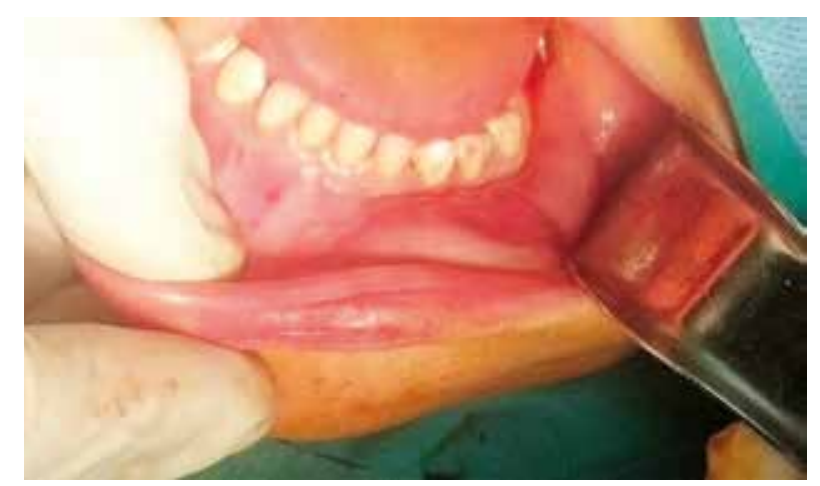

Fig. 1. Bony hard swelling causing obliteration of the vestibule of left side of mandible, extending from the right deciduous lateral incisor to the left deciduous second molar region 
A

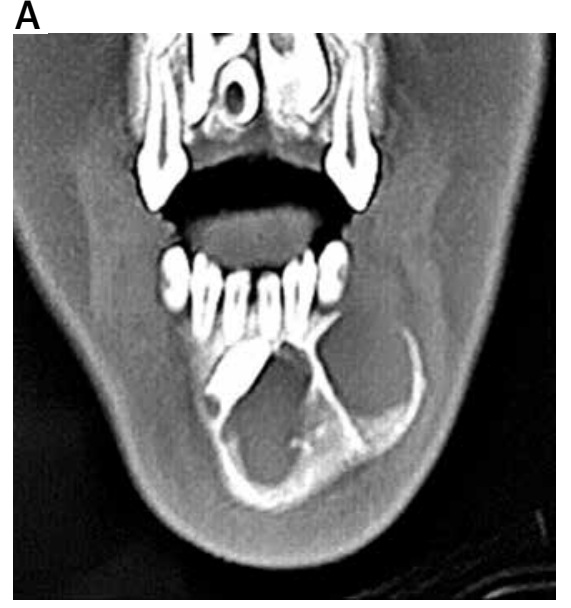

B

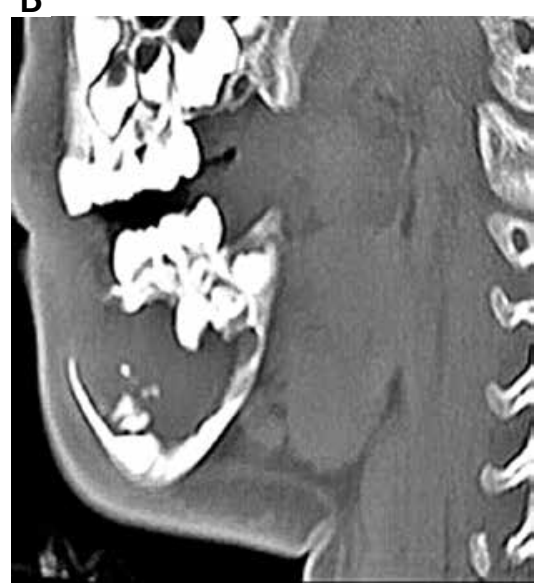

C

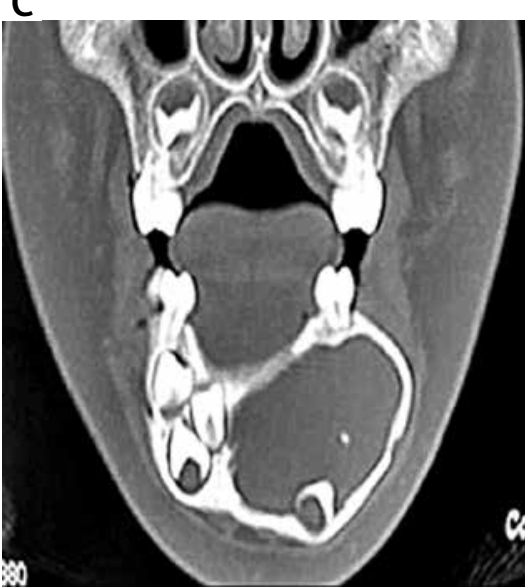

Fig. 2. A) The CT revealed a well-defined multilocular radiolucent lesion extending from the lower right deciduous lateral incisor to the right deciduous second molar region. B) Bucco-lingual expansion, perforation and thinning of the buccal plate were observed. The lesion also showed small calcified areas. C) Multiple impacted permanent teeth were noted
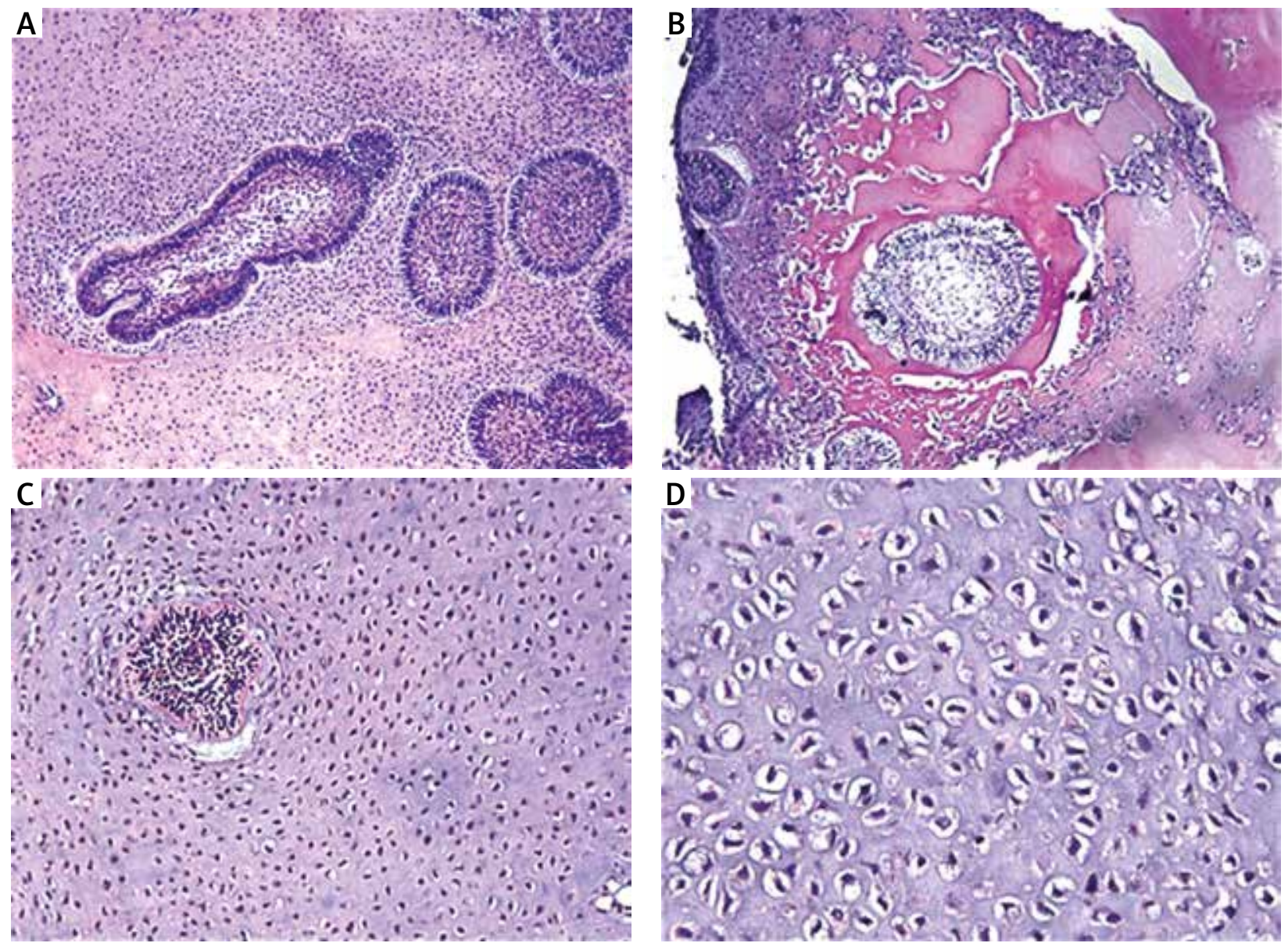

Fig. 3. A) Multiple follicles of proliferating odontogenic epithelium with the surrounding primitive ectomesenchyme showed increased cellularity simulating the dental papillae. B) Areas showing dentin formation and enamel spaces (hematoxylin-eosin, original magnification 100×). C, D) Areas with cartilage formation were also observed. These areas exhibited increased maturation as they progressed further away from the epithelial follicles, showing hypercellularity and some binucleated cells (hematoxylin-eosin, original magnification: C) 200×, D) 400x)

bony hard swelling causing obliteration of the vestibule of the left side of the mandible, extending from the right deciduous lateral incisor to the left deciduous second molar region, measuring approximately approximately $7 \times 4 \mathrm{~cm}$ in size. The swelling caused buccal and lingual cortical expansion and the buccal plate was perforated in some areas (Fig. 1).
The CT revealed a well-defined multilocular radiolucent lesion extending from the lower right deciduous lateral incisor to the left deciduous second molar region causing bucco-lingual expansion and thinning of the buccal plate. The lesion also showed small calcified areas and multiple impacted permanent teeth (Fig. 2). Based on the clinical 

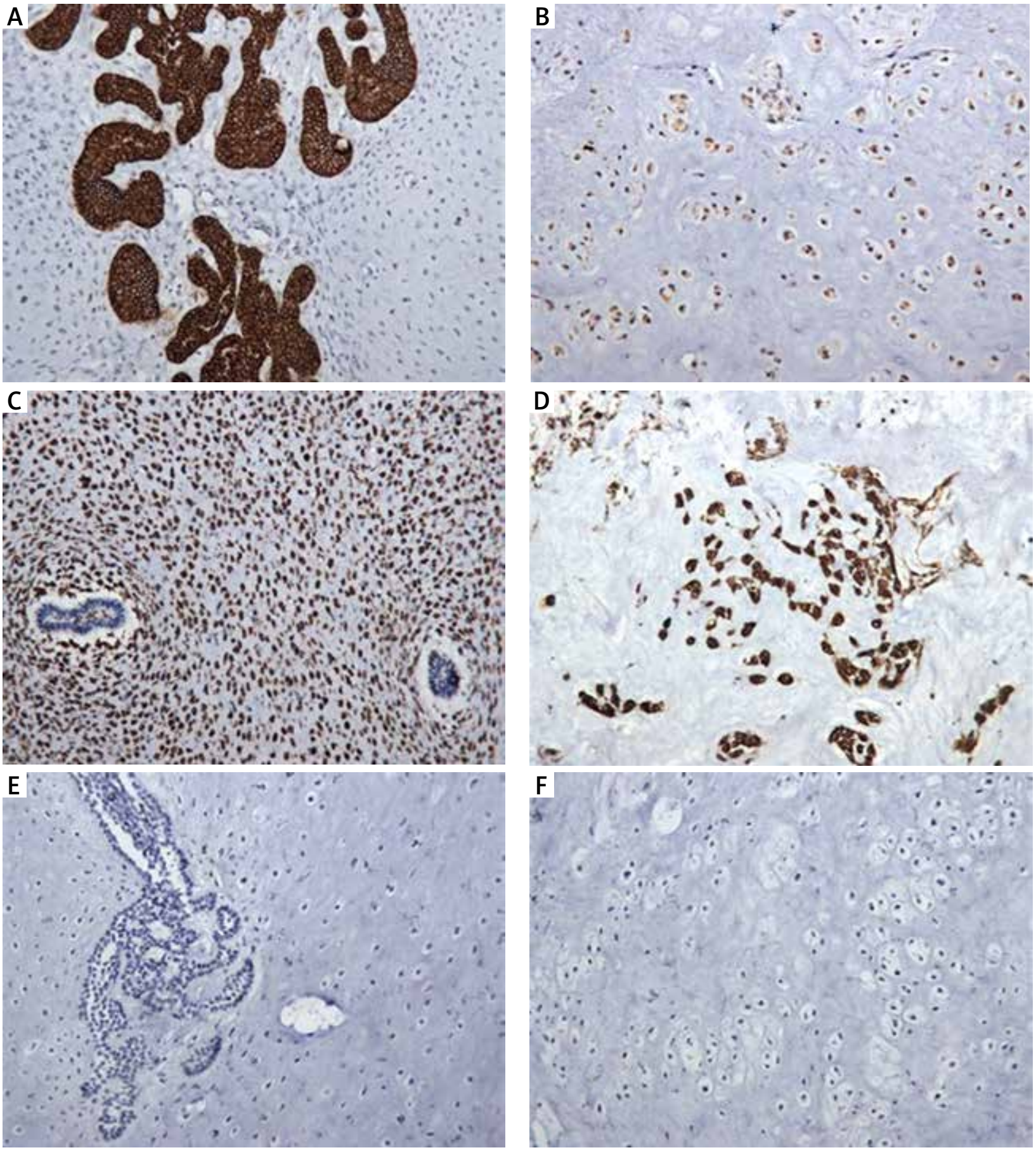

Fig. 4. A) Immunohistochemical examination revealed that all epithelial components showed strong diffuse reactivity for cytokeratin. B) The chondroid tissue also showed cytokeratin expression. C) Vimentin expression was detected in both the stromal ectomesenchymal component (D) and in the chondroid tissue (E \& F) S-100 protein showed negative expression in all components (original magnification $200 \times$ )

and radiographic findings, a presumptive preoperative diagnosis of odontogenic tumour was made with differential diagnosis of AFO, calcifying epithelial odontogenic tumour and calcifying odontogenic cyst.

Incisional biopsy was performed and the specimen was sent to the oral and maxillofacial pathology department for histopathological analysis. Macroscopically, the specimen was white in colour, measured $3 \times 2.3 \times 2 \mathrm{~cm}$ in great- est dimension and was firm in consistency with irregular surface.

Microscopically, the lesion showed multiple follicles of proliferating odontogenic epithelium. The odontogenic epithelium showed peripheral tall columnar cells exhibiting nuclear palisading, hyperchromatism, and reversed polarity. The central cells were loosely arranged and angular to spindle in shape (Fig. 3A). The surrounding primitive ec- 
tomesenchyme showed increased cellularity simulating the dental papillae with areas showing dentin formation (Fig. 3B). Extensive areas with cartilage formation were also observed, showing hypercellularity and some binucleated cells. The cartilaginous areas exhibited increased maturation as they progressed further away from the epithelial follicles (Fig. 3C, D). Immunohistochemical examination revealed that all epithelial components showed strong diffuse reactivity for cytokeratin (Fig. 4A). The chondroid tissue also showed cytokeratin expression (Fig. 4B). Vimentin expression was detected in both the stromal ectomesenchymal component (Fig. 4C) and in the chondroid tissue (Fig. 4D). S-100 protein showed negative expression in all components (Fig. 4E, F).

The lesion was treated by complete surgical excision and the excisional biopsy was examined confirming the diagnosis. The patient was followed-up for 6 months without any signs of recurrence.

\section{Discussion}

Ameloblastic fibroma is a rare, benign, mixed odontogenic tumour, comprising $1.5-4.5 \%$ of all odontogenic tumours [3], and is characterised by proliferating odontogenic epithelium embedded in a cellular ectomesenchymal tissue resembling dental papilla, with no hard dental tissue formation [4]. Lesions formed of similar components, but characterized by deposition of dentin alone or dentin plus enamel, are termed AFD and AFO, respectively [2]. Cahn and Blum in 1952 [5] stated that AF entitled as the least histologically differentiated neoplasm that develops to a moderately differentiated form to AFO and eventually into odontoma. Nevertheless, this theory is not widely accepted, and there is much debate regarding the relationship and nature of these lesions as either being the same in a continuum representing different stages of evolution or considered to be separate pathological entities [6, 7].

Ameloblastic fibro-odontoma is rare, and comprise only 1 to $3 \%$ of all odontogenic tumours. It occurs predominantly in children and young adults, with an equivalent incidence observed in both upper and lower jaws and no reported gender predilection [8].

Radiographic examinations of AFO usually show a well-demarcated unilocular or multilocular radiolucent lesion with radiopaque foci of varying amounts and size. Microscopic evaluation of AFO reveals cords, strands and islands of odontogenic epithelium submerged in primitive connective tissue and formation of hard material consisting of foci of enamel and dentine [9]. As far as we know, this is the first reported case to show evidence of cartilage deposition. Whether this tissue represents actual cartilage formation, or whether it simply represents a metaplastic process resulting in a cartilage like tissue remains to be elucidated. However, the negative S100 staining and the positive cytokeratin staining within the tissue suggests that this is more of a chondroid tissue rather than actual cartilage. Ameloblastic fibro-odontoma are usually treated by conservative enucleation with curettage.
The authors declare no conflict of interest.

\section{References}

1. El-Naggar KA, Chan KCJ, Grandis RJ, Takata T, Slootweg JP. WHO Classification of Head and Neck Tumours. $4^{\text {th }}$ edition, IARC 2017; 2-17.

2. Kramer IR, Pindborg JJ, Shear M. Histological typing of odontogenic tumours. Springer Science \& Business Media 1992.

3. Cohen DM, Bhattacharyya I. Ameloblastic fibroma, ameloblastic fibro-odontoma, and odontoma. Oral Maxillofac Surg Clin North Am 2004; 16: 375-84.

4. Munde AD, Karle RR, Kale UB. Ameloblastic fibroma in one-year-old girl. J Oral Maxillofac Pathol 2013; 17: 149.

5. Cahn LR, Blum T. Ameloblastic odontoma: case report critically analyzed. J Oral Surg 1952; 10: 169-70

6. Slootweg PJ. An analysis of the interrelationship of the mixed odontogenic tumours - ameloblastic fibroma, ameloblastic fibro-odontoma, and the odontomas. Oral Surg Oral Med Oral Pathol 1981; 51: 266-76.

7. Gardner DG. The mixed odontogenic tumours. Oral Surg Oral Med Oral Pathol. 1984; 58: 166-8.

8. Sreenath G, Reddy I, Sreenivasreddy P. Ameloblastic fibro-odontome (AFO) of the mandible: A case report. J Clin Diagn Res 2014; 8: $260-2$.

9. Nelson BL, Thompson LD. Ameloblastic fibro-odontoma. Head Neck Pathol 2014; 8: 168-70.

\section{Address for correspondence}

Noura Aly

Faculty of Dentistry, Cairo University

11 EL-Saraya St. Manial

11553 Cairo, Egypt

e-mail: noura.ali@dentistry.cu.edu.eg

Submitted: 25.02 .2018

Accepted: $\quad 3.03 .2018$ 